\section{Wnt signaling drives WRM-1/ $\beta$-catenin asymmetries in early C. elegans embryos}

\author{
Kuniaki Nakamura, ${ }^{1}$ Soyoung Kim, ${ }^{1}$ \\ Takao Ishidate, ${ }^{1}$ Yanxia Bei, ${ }^{1}$ Kaming Pang, ${ }^{1}$ \\ Masaki Shirayama, ${ }^{1}$ Chris Trzepacz, ${ }^{1}$ \\ Daniel R. Brownell, ${ }^{2}$ and Craig C. Mello ${ }^{1,2,3}$
}

${ }^{1}$ Program in Molecular Medicine, University of Massachusetts Medical School, Worcester, Massachusetts 01605, USA; ${ }^{2}$ Howard Hughes Medical Institute

$\beta$-Catenin regulates cell adhesion and cellular differentiation during development, and misregulation of $\beta$-catenin contributes to numerous forms of cancer in humans. Here we describe Caenorhabditis elegans conditional alleles of mom-2/Wnt, mom-4/Tak1, and wrm$1 / \beta$-catenin. We use these reagents to examine the regulation of WRM-1/ $\beta$-catenin during a Wnt-signaling-induced asymmetric cell division. While WRM-1 protein initially accumulates in the nuclei of all cells, signaling promotes the retention of WRM-1 in nuclei of responding cells. We show that both PRY-1/Axin and the nuclear exportin homolog IMB-4/CRM-1 antagonize signaling. These findings reveal how Wnt signals direct the asymmetric localization of $\boldsymbol{\beta}$-catenin during polarized cell division.

Supplemental material is available at http://www.genesdev.org.

Received April 14, 2005; revised version accepted June 9, 2005.

Wnt proteins are secreted signaling molecules important in numerous developmental events in animals (Cadigan and Nusse 1997; Zou 2004). They signal through multipass membrane receptors of the Frizzled family, through coreceptor complexes composed of Frizzled receptors and members of the Arrow LRP5/6 protein family, and through members of the RYK family of receptor tyrosine kinase-related proteins (Yoshikawa et al. 2003; Inoue et al. 2004; Lu et al. 2004). $\beta$-Catenin is a multifunctional protein, with a role in cell adhesion mediated through its interaction with E-cadherin, and a role in transcription that is mediated through interactions with members of the TCF/LEF transcription factor family (Bullions and Levine 1998; Nelson and Nusse 2004). One well-established paradigm for Wnt-mediated transcriptional regulations involves the signaling-dependent down-regulation of a $\beta$-catenin destruction complex consisting of Axin, GSK-3 $\beta$, and APC, thus increasing the levels of $\beta$-catenin available for nuclear translocation and tran-

[Keywords: $\beta$-Catenin; wrm- 1 ; wnt; nuclear export; asymmetric cell division; C. elegans]

${ }^{3}$ Corresponding author.

E-MAIL Craig.Mello@umassmed.edu; FAX (508) 856-2950.

Article and publication are at http://www.genesdev.org/cgi/doi/10.1101/ gad.1323705. scriptional activation (Bullions and Levine 1998; Willert and Nusse 1998; Polakis 2000). However, Wnt signaling can also influence the polarity of responding cells (Bei et al. 2002; Lyuksyutova et al. 2003; Gong et al. 2004), and at present there is little insight into how Wnt signaling induces cytoskeletal asymmetries and directs the asymmetric activation of $\beta$-catenin in the daughters of responding cells.

Caenorhabditis elegans provides an ideal system for analyzing the role of Wnt signaling in polarized cell divisions. In addition to powerful genetic tools available in this animal, signaling events can be analyzed at the level of the individual cells involved in signaling. For example, at the four-cell stage of embryogenesis, a ventral cell called EMS receives a signal from the posterior-most cell, P2. Signaling from P2 orients the EMS division axis onto the anterior-posterior $(\mathrm{a} / \mathrm{p})$ axis of the embryo (Bowerman and Shelton 1999) and induces an unequal division that gives rise to one anterior mesodermal precursor and one posterior endodermal precursor (Goldstein 1993).

P2/EMS signaling involves multiple inputs, including at least two parallel cell-surface receptor-mediated pathways: the Wnt-Frizzled pathway (Rocheleau et al. 1997; Thorpe et al. 1997) and a Src-mediated receptor tyrosine kinase signaling pathway (Bei et al. 2002). Transcriptional outputs downstream of P2/EMS signaling depend on a $\beta$-catenin homolog, WRM-1 (pronounced "worm1 "). WRM-1 functions in concert with the conserved Ser/ Thr kinase, LIT-1, to inhibit POP-1, a TCF/LEF-related transcriptional repressor (Ishitani et al. 1999; Meneghini et al. 1999; Rocheleau et al. 1999). In studies using cultured vertebrate cells, interactions between WRM-1 and LIT-1 activate the LIT-1 kinase, leading to LIT-1-dependent phosphorylation of WRM-1, LIT-1, and POP-1, and resulting in a net nuclear export of POP-1 protein (Rocheleau et al. 1999). Recent in vivo studies suggest that signaling leads to the nuclear accumulation of LIT-1 and activates the nuclear export of POP-1 through a mechanism dependent on PAR-5, a 14-3-3 homolog, and IMB-4, a homolog of the $\beta$-importin-nuclear transport factor CRM-1 (Lo et al. 2004).

Despite this progress, many gaps remain in our understanding of how P2/EMS signaling directs EMS spindle orientation and how this signaling leads to unequal POP-1 nuclear levels in the daughters of EMS. In particular, although the WRM-1/ $\beta$-catenin protein appears to represent a nexus for coordinating signals from the membrane and facilitating their transduction to the nucleus, little is known about whether and how WRM-1 activity or localization is regulated during signaling.

Here we analyze the regulation of WRM-1 during the EMS cell division. We show that WRM-1::GFP initially enters the nucleus at the beginning of telophase in all cells but is exported in signal-nonresponding cells. Nuclear export requires the CRM-1-exportin homolog, IMB-4, and Ran-related molecules, including Ran-3/ RCC1 and Ran-5/RanBP3. Wnt signaling promotes the nuclear maintenance and/or continued accumulation of WRM-1 in daughter cells proximal to the polarizing signal. Our findings support a model for Wnt signaling-dependent polarized cell division in which signaling controls the nuclear accumulation of $\beta$-catenin. 


\section{Results and Discussion}

Isolation of temperature-sensitive mutants defective in polarity signaling

To better understand the mechanism of Wnt signaling in C. elegans, we conducted an unbiased screen for temperature-sensitive (ts) mutants defective in polarity signaling (see Materials and Methods). Among 4000 ts mutations that disrupt C. elegans embryogenesis, we found 30 mutants with defects in P2/EMS signaling. These include nine alleles of mom-2/Wnt, 12 alleles of lit-1/Nlk, four alleles of mom-4/MAPKKK, and a single allele of wrm-1/ $\beta$-catenin (Fig. 1). While a ts allele of lit-1 was described previously (Kaletta et al. 1997), the mom-2, mom-4, and wrm-1 mutants are the first ts alleles of these genes and provide useful tools for analyzing the function of these genes in embryogenesis and during later development. When L4 or adult wrm-1(ne1982) animals are shifted to nonpermissive temperature, the resulting embryos exhibit a penetrant loss of endoderm phenotype identical to the previously described wrm1(RNAi) phenotype (data not shown). Temperature-shift studies indicate that WRM-1 is required (1) in early embryos for specification of endoderm in response to P2/ EMS signaling, (2) after the four-cell stage for embryonic viability and morphogenesis, and (3) during larval development for fertility and for the production of a functional vulva (Supplementary Table 1; data not shown).

\section{Signal-dependent asymmetric localization} of WRM-1::GFP

To follow the localization of WRM-1, we rescued wrm1(ne1982) with an N-terminal GFP-tagged transgene. This transgene provided nearly $100 \%$ rescue of all of the phenotypes associated with wrm-1(ne1982) and 80\%$90 \%$ rescue of embryos produced by animals homozy- gous for a presumptive-null, deletion allele of wrm1(tm514). Consistent with its role in P2/EMS signaling, WRM-1::GFP levels were high in the nucleus of the E blastomere at the eight-cell stage (Fig. 2A). However, a time course of images revealed that WRM-1::GFP is at first localized similarly in both newly formed nuclei at the beginning of telophase of the EMS cell division (Fig. 2A, top panels, arrowheads). Furthermore, even signalnonresponsive cells, including cells present at the one-, two- and four-cell stages, exhibit nuclear accumulation of WRM-1 at the beginning of telophase (data not shown). During the EMS cell division, the nuclear signal in MS grows weaker, while in E, the nuclear WRM$1::$ GFP levels become higher (Fig. 2A). A high WRM$1::$ GFP nuclear level during and after cytokinesis was only observed in signal-responding cells, including numerous posterior descendants of later a/p divisions (Fig. $2 \mathrm{~B}$; data not shown). In addition to its change in nuclear levels, we also observed a cell cycle- and signal-dependent change in the localization of WRM-1 protein at the cell cortex and cell-cell contact sites (Fig. 2A,B, arrows; see below).

\section{Regulation of WRM-1 nuclear localization}

The above findings indicate that the nuclear distribution of WRM-1 may be regulated by signaling. The observation that WRM-1 enters the nucleus in all cells but is maintained there only in signal-responding cells prompted us to ask which of the known nuclear export factors are required for WRM-1 nuclear export. We found that IMB-4, a homolog of human exportin chromosome maintenance region-1 protein (CRM-1) (Fornerod et al. 1997; Fukuda et al. 1997), as well as several of its conserved cofactors, including RAN-1/Ran, RAN-3/RCC1/ RanGEF, and RAN-5/RanBP3, all appear to be critical for WRM-1 nuclear export. The depletion of these factors resulted in a dramatic accumulation of WRM-1::GFP in
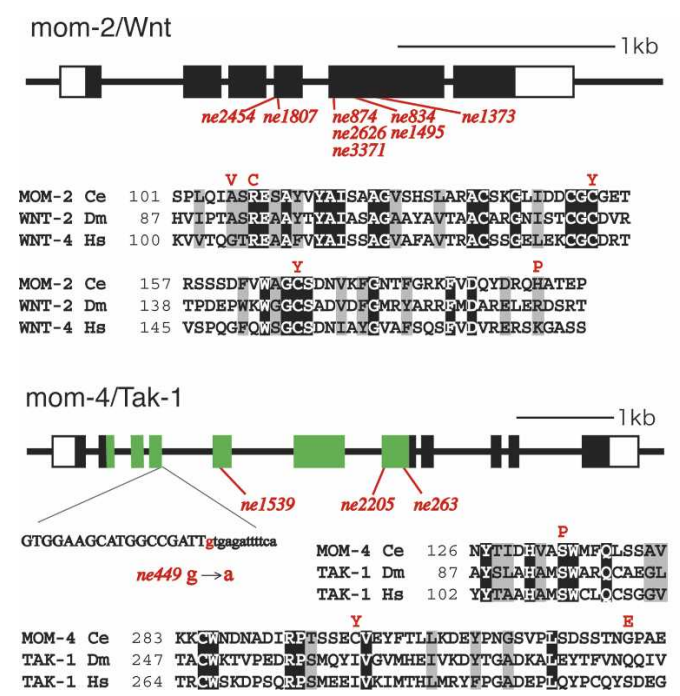
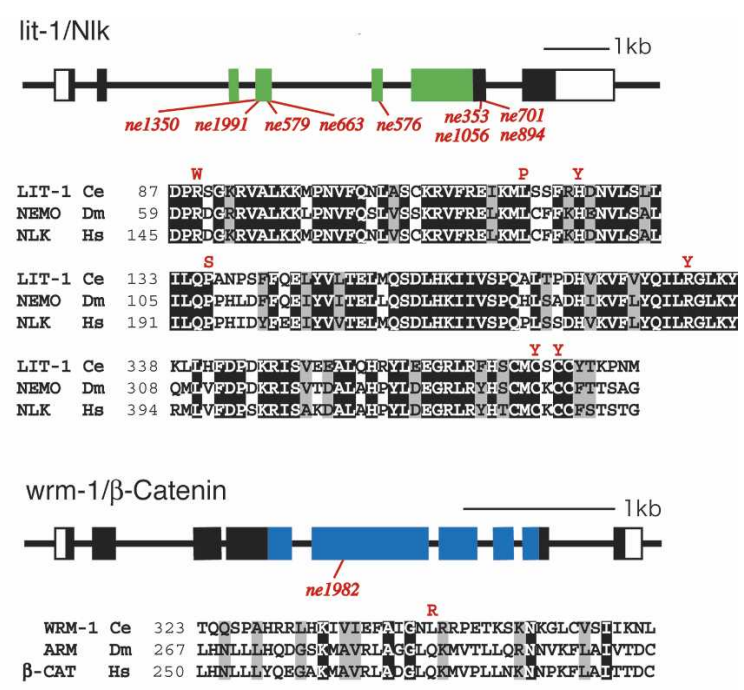

Figure 1. Temperature-sensitive (ts) mutants defective in P2/EMS signaling. Schematic diagrams and alignments for four P2/EMS signaling genes. Ts-genetic lesions and their allele names are shown below the genomic box/line-exon/intron structures. Alleles with identical lesions are indicated together in columns. In mom-4 and lit-1, the kinase domains are indicated in green; in wrm-1, the arm-motif region is indicated in blue. White and black boxes indicate noncoding and coding regions, respectively. A splice donor site lesion is indicated below the structure of mom-4 with the intron sequence shown in lowercase. Amino acid substitutions for each distinct lesion are shown in red type above alignments for the corresponding region in each protein. The alignments compare each C. elegans (Ce) protein to Drosophila melanogaster (Dm) and Homo sapiens (Hs) homologs, as indicated. 
A

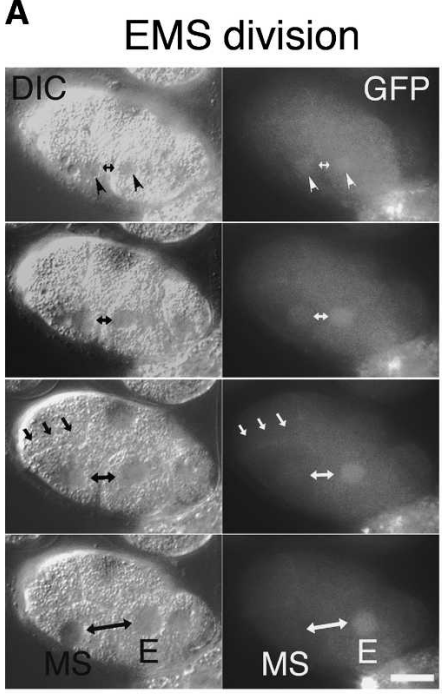

C
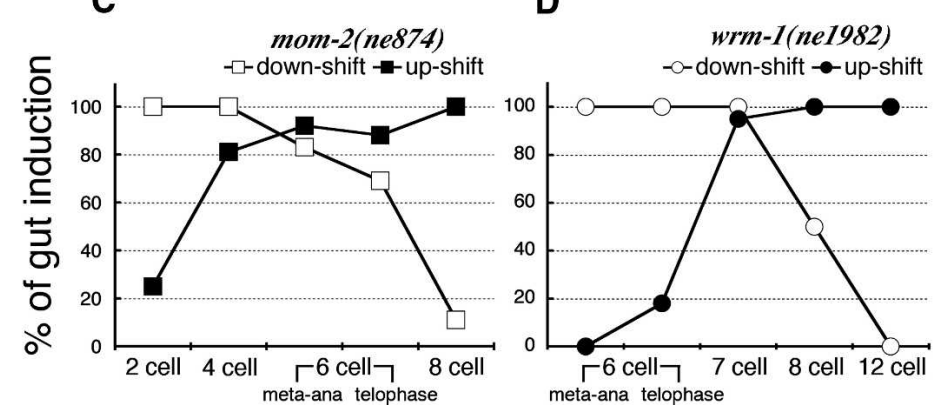

Figure 2. Cell cycle-dependent asymmetric localization of WRM-1 : GFP. $(A, B)$ Nomarski (left columns) and fluorescence micrographs (right columns) of wildtype WRM-1::GFP transgenic animals taken during EMS and ABal cell divisions. (Top panels) White arrowheads indicate the faint, initially similar levels of WRM-1::GFP in early telophase nuclei. Double-headed arrows indicate the nuclei of anterior and posterior sisters. White arrows indicate the cortical localization of WRM-1 ::GFP. Black arrows indicate the corresponding structures in each Nomarski image. In this and subsequent figures, the bars indicate $10 \mu \mathrm{m}$ and anterior is to the left. $(C, D)$ Graphic depictions of the temperature-sensitive (ts) periods for gut induction in mom-2(ne874) $(C)$ and wrm-1(ne1982ts) $(D)$. The embryos were either up-shifted (black squares or circles) or down-shifted (white squares or circles) at the indicated cell stage $(X$-axis $)$, and incubated further to score for gut induction ( $Y$-axis). Shifts were done at two times during the EMS cell cycle: metaphase/anaphase (meta-ana) and telophase (as indicated).

the nuclei of all cells in the early C. elegans embryo. For example, we found that, during telophase in imb-4, ran3, and ran-5 RNAi embryos, WRM-1::GFP levels continued to increase in all cells, including both daughters of EMS (Supplementary Fig. 1). These findings suggest that IMB-4-dependent nuclear export may function as a default pathway that lowers the nuclear levels of WRM-1 in the absence of signaling.

We next asked whether any of the known P2/EMS signaling factors influence the nuclear localization of WRM-1 ::GFP. In mom-2/Wnt mutant embryos, and in several other mutants defective in polarity signaling, we found that, while the initial nuclear accumulation of WRM-1::GFP at telophase was unaffected, the subsequent maintenance of WRM-1 levels was reduced or abolished in the E nucleus and in other normally signalresponsive cells (Fig. 3; data not shown). As in wild-type embryos, inhibition of imb-4 in mom-2 and several other signaling mutants resulted in equal and high levels of WRM-1::GFP staining in all nuclei (Fig. 3; Supplementary Figs. 1-3). These observations suggest that signaling acts late in the cell cycle to maintain high nuclear levels of WRM-1 in responding cells, and may do so, directly or indirectly, by counteracting IMB-4/CRM-1-dependent nuclear export. Consistent with the notion that signaling acts late in the cell division cycle of responding cells, we found that the temperature-sensitive period (tsp) for mom-2(ne874) is late in the EMS cell division cycle (Fig. 2C), and the tsp for wrm-1(ne1982) is shortly after the EMS cell division (Fig. 2D). This latter finding suggests that the WRM-1(ne1982) mutant protein may undergo proper regulation during the EMS division but is unable to execute its downstream functions in the E nucleus when maintained at the nonpermissive temperature.

The initial accumulation of WRM-1::GFP in telophase nuclei was unaltered in nearly all of the strains tested, with the notable exception of the lit-1 and mom-4 mutants. In lit-1 mutants, the GFP signal was strongly excluded from the nucleus in all cells (Fig. 3; Supplementary Fig. 2). This nuclear exclusion was not dependent on IMB-4 (Fig. 3; Supplementary Fig. 3). Similarly, MOM-4 appeared to be required generally for nuclear accumulation but, consistent with the lower penetrance of its signaling defect (Shin et al. 1999), exhibited a less complete nuclear exclusion of WRM-1::GFP (Supplementary Figs. 2, 3). Thus, LIT-1 and MOM-4 appear to play a general role in permitting the initial nuclear accumulation of WRM-1, while IMB-4 may function independently to promote nuclear export.

In other organisms, TCF-related factors require $\beta$-catenin to activate transcription, and the binding of $\beta$-catenin to TCF in the nucleus has been implicated in the nuclear retention of $\beta$-catenin (Tolwinski and Wieschaus 2001, 2004; Townsley et al. 2004). In C. elegans, the TCF-related protein POP-1 is negatively regulated by WRM-1, leading to nuclear export of POP-1 (Lin et al. 1998). Surprisingly, WRM1 levels in the nucleus of E were reduced in the absence of POP-1 (Fig. 3; Supplementary Fig. 3), suggesting that interactions with POP-1 influence the nuclear localization of WRM-1 despite the opposite nuclear asymmetries for these two proteins. In several systems, a complex consisting of Axin, APC, and GSK-3 $\beta$ negatively regulates $\beta$-catenin stability, and these molecules have also been implicated in the nuclear export of $\beta$-catenin in a mammalian-cell-culture system (Rosin-Arbesfeld et al. 2003; Cong and Varmus 2004). However, in contrast to other systems, both APR-1/APC and GSK-3/GSK-3 $\beta$ appear to positively influence endoderm specification in C. elegans (Rocheleau et al. 1997; Schlesinger et al. 1999). Consistent with these genetic data, we found that WRM-1 nuclear levels are reduced in the E blastomere in both apr-1(RNAi) and gsk-3(RNAi) embryos (Fig. 3; Supplementary Fig. 3). Inhibition of nuclear export via imb-4(RNAi) in apr-1- and gsk-3-depleted embryos resulted in nuclear WRM-1 accumulation similar to that seen in imb-4(RNAi) singly-depleted embryos (Fig. 3, right panels). Thus, WRM-1 protein appears to be stable, 


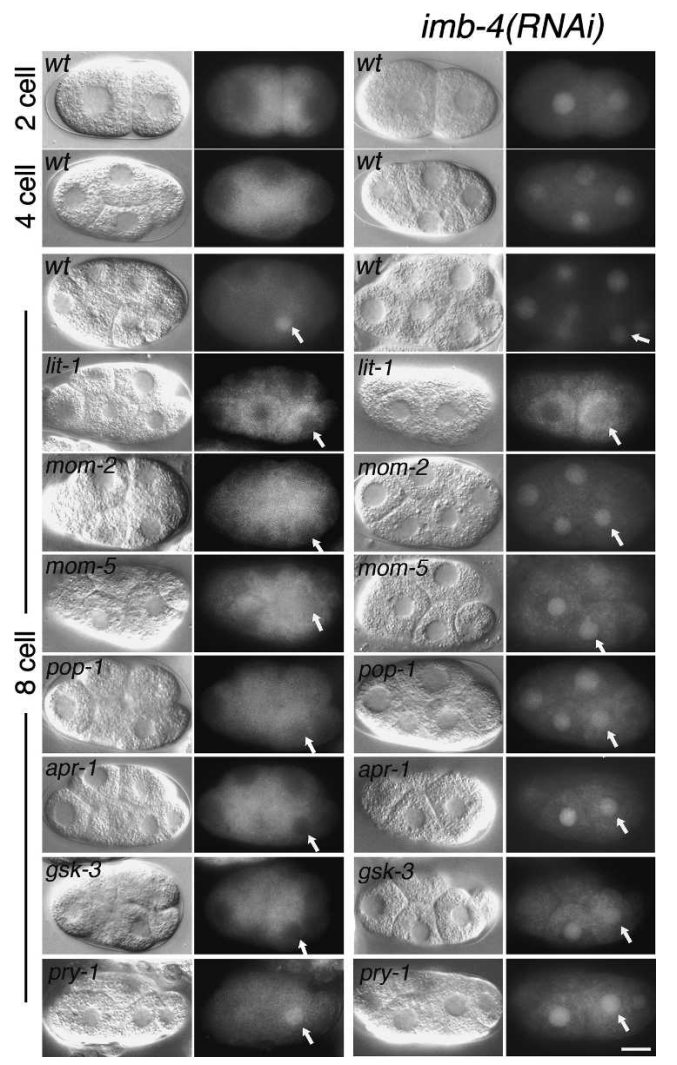

Figure 3. Genetics of WRM-1 nuclear accumulation. Nomarski and WRM-1::GFP-fluorescence micrographs of interphase transgenic embryos. The cell stage and genotypes are as indicated. Embryos in the right two columns are genotypically identical to those in the left two columns but are also subject to imb-4(RNAi). The alleles used are lit-1(ne1991), mom-2(ne874), mom-5(zu193), apr1(RNAi), pop-1(RNAi), gsk-3(RNAi), and pry-1(mu38). The E nucleus is indicated with a white arrow in each eight-cell-stage embryo.

but fails to be retained in the nuclei of apr-1 and gsk-3 mutant embryos.

\section{pry-1 and imb-4 antagonize Wnt signaling}

Mutations in the C. elegans Axin-related gene pry-1 (Korswagen et al. 2002) exhibit a subtle but reproducible effect on WRM-1 protein accumulation. In pry-1 mutants, WRM-1 consistently accumulates to slightly higher levels in nuclei of signal-responding cells (Fig. 3). Interestingly, we found that pry-1 mutants can suppress wrm-1(ne1982) to viability. Approximately 30\% of the embryos produced by wrm-1(ne1982) pry-1(mu38) double-mutant animals are viable at the restrictive temperature of $23^{\circ} \mathrm{C}(n=300)$, as opposed to only $0.1 \%$ of embryos produced by wrm-1(ne1982) single mutants $(n>1000)$. This suppression of wrm-1 included partial suppression of later embryonic, as well as larval and fertility defects, associated with the wrm-1(ne1982) mutant phenotype, suggesting that PRY-1 plays a significant role in negatively regulating WRM-1 protein at all stages (data not shown). We found that pry-1(mu38) exhibits a similar level of suppression of lit-1(ne1991) mutants (data not shown). These results suggest that C. elegans PRY-1/Axin negatively regulates WRM-1, whereas APR$1 / \mathrm{APC}$ and GSK-3 $\beta$ appear to be positive regulators, at least during the EMS division.
The strong suppression of wrm-1(ne1982) by pry1(mu38) mutants was surprising considering the relatively subtle increase in WRM-1 nuclear levels observed in pry-1 mutants and the lack of detectable P2/EMS signaling defects in pry-1 single mutants. Thus, it is possible that PRY-1 negatively regulates WRM-1 activity rather than WRM-1 localization. However, signaling does appear to be extremely sensitive to WRM-1 protein levels. For example, we found that the introduction of the WRM-1::GFP transgene into the mom-2(ne874) mutant strain was sufficient to rescue the strain to viability. Conversely, reducing wrm-1 activity in mom-2(ne874) or mom-5(zu193) mutant backgrounds dramatically increased the gut induction defects in these mutants (data not shown).

Conceivably, the maintenance of high WRM-1 nuclear levels could merely be correlated with signaling but not be required for the outcome of signaling. To examine this possibility, we asked if artificially increasing WRM-1 nuclear levels by using RNAi to disrupt the IMB-4-dependent nuclear transport machinery could suppress the loss of endoderm defect in mom-2/Wnt. We found that the percentage of embryos with endoderm produced by mom-2(ne874) homozygous mutant mothers increased from $40 \%(n=277)$ to $75 \%(n=268)$ after imb-4(RNAi), suggesting that the nuclear-localization pathway is indeed important for controlling the outcome of signaling (Supplementary Fig. 4). This suppression was dependent on the activities of both WRM-1 and LIT-1 (Supplementary Fig. 4). Because IMB-4 is also required for POP-1 export (Lo et al. 2004), it is interesting to note that WRM-1-dependent endoderm differentiation is restored in the E blastomere despite the existence of a high level of nuclear POP-1. These and other observations (Maduro et al. 2002) suggest that, in addition to controlling the nuclear localization of POP-1, signaling via WRM-1 also regulates the activity of the POP-1 protein in $\mathrm{E}$.

\section{A model for WRM-1 regulation during polarized cell division}

While the above studies suggest that signaling regulates WRM-1 nuclear localization, they do not explain how signaling events at the cell cortex are coupled to the asymmetric nuclear accumulation of WRM-1. A possible insight into the connection between cortical and nuclear signaling events comes from preliminary findings on the cortical localization of WRM-1. In the course of our studies, we noticed a faint localization of WRM-1::GFP to the cell cortex during each mitosis (Fig. 2A,B, arrows). Interestingly, in the EMS cell, WRM-1::GFP is lost along the posterior cortex proximal to the signaling cell P2, while staining is maintained along the anterior cortex of the dividing EMS cell (Supplementary Fig. 5). This cortical localization is also visible at later stages (data not shown) and in developing larvae (see Takeshita and Sawa 2005). Our preliminary studies suggest that MOM-5/ Frizzled is required for cortical association, while cortical release correlates with signaling via MOM-2/Wnt (Supplementary Fig. 6). Although these observations require further investigation, they suggest an interesting model that could explain how signaling at the cortex could drive nuclear WRM-1 asymmetries. Importantly, this model could also explain the difference between the penetrance of the endoderm defects seen in mom-2/Wnt mutants ( $60 \%$ gutless) and mom-5/Fz mutants (only 
$5 \%$ gutless), and the surprising finding that the lower penetrance gutless phenotype of mom-5 is epistatic to mom-2 (Rocheleau et al. 1997; Bei et al. 2002).

According to this model (Fig. 4), P2/EMS signaling alters the affinity of WRM-1 for the posterior cortex of EMS and simultaneously activates WRM-1 for downstream signaling. This activation could be direct (e.g., by phosphorylation of WRM-1) or indirect (e.g., by modification of a WRM-1-interacting protein such as LIT-1). For simplicity in this discussion, we will consider the direct activation model. At steady state, only a small percentage of WRM-1 protein localizes at the cortex and this level drops during signaling, suggesting that cortical association may reflect a dynamic process that is modulated by signaling. Cortical signaling events also ensure that the mitotic apparatus of the cell is oriented such that division produces one nucleus that is more proximal to the posterior cortex and thus exposed to higher concentrations of an activated and less cortically associated form of WRM-1. At the beginning of telophase, WRM-1 accumulates in both nascent nuclei via a mechanism that depends on the kinases MOM-4 and LIT-1. During late telophase, and shortly after cytokinesis, IMB-4/ CRM-1-dependent export begins to reduce WRM-1 nuclear levels in MS. However, in E, the signal-dependent release of an activated form of WRM-1 from the cortex induces a net nuclear retention of WRM-1. Finally, retention of WRM-1 in the nascent E nucleus correlates with a simultaneous CRM-1-dependent nuclear export of POP-1.

This model explains the phenotypic differences between mom-2 and mom-5 mutants. In mom-2 mutants, MOM-5 sequesters WRM-1 at the posterior cortex, reducing WRM-1 nuclear retention in E, and resulting in the higher penetrance of the mom-2 endoderm defect. In mom-5 mutants or in mom-2; mom-5 double mutants, signaling from P2 via the parallel SRC-1 tyrosine kinase pathway can activate WRM-1, which is then free to enter the nucleus and promote POP-1 nuclear export. Since SRC-1 has little effect on WRM-1 localization (data not shown), our findings suggest that SRC-1 may instead alter WRM-1 activity.

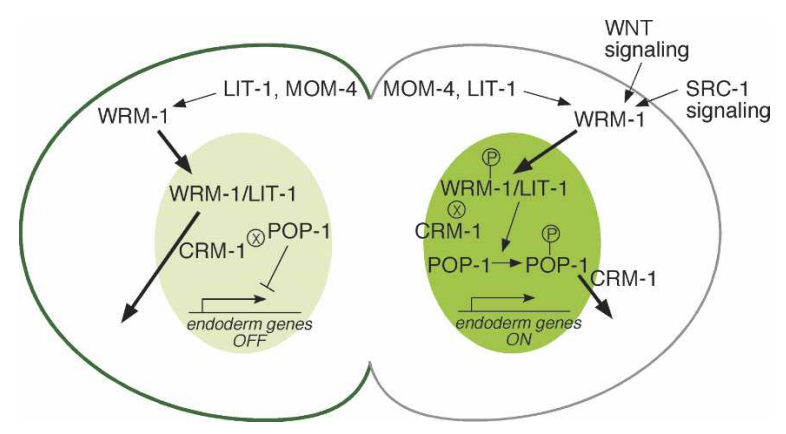

Figure 4. A model for asymmetric localization of WRM-1::GFP. Schematic diagram of polarity signaling in the EMS cell at telophase. WRM-1 protein is retained in the posterior E nucleus (dark green) and exported from the MS nucleus (lighter green). Conversely, WRM-1 is preferentially retained, in a MOM-5/Fz-dependent manner, at the anterior cortex (dark-green line) and released at the posterior cortex (gray line). Arrows and bars between signaling components depict activating and inhibitory genetic interactions, respectively. Signaling from Wnt and Src leads to phosphorylation of WRM- 1 and POP-1 (indicated by the P within a circle). The $\otimes$ symbol adjacent to WRM-1 (in E) and POP-1 (in MS) indicates reduced CRM1-dependent export. Bold arrows indicate nuclear entry or export.
The details of the mechanism that drives the reciprocal nuclear accumulation of WRM-1 and POP-1 are still not clear. Our finding that the nuclear accumulation of WRM-1 partially depends on POP-1 suggests that WRM-1 and POP-1 may directly compete for nuclear export factors or nuclear/cytoplasmic retention sites. For example, WRM-1-dependent phosphorylation of POP-1 (Fig. 4) might increase the affinity of POP-1 for CRM-1, perhaps by promoting the interaction of POP-1 with PAR-5/14-3-3. This could lead to a direct competition that displaces WRM-1 from the export machinery in responding cells. Alternatively, signaling may alter the relative affinity of WRM-1 and/or POP-1 for binding to mutually exclusive partners in the nucleus or in the cytoplasm, causing a simultaneous and codependent shift in the net balance of their nuclear/cytoplasmic retention.

In summary, we have analyzed the regulation of a $\beta$-catenin homolog, WRM-1, during a polarized cell division in C. elegans. The findings presented here suggest that WRM-1 is subject to regulation at multiple levels, and begin to place the surprising genetic complexity of P2/EMS signaling into a cell-biological context. Furthermore, our findings suggest that Wnt signaling can control the nuclear accumulation of $\beta$-catenin and may also influence its cortical distribution. These modes of regulation may be of particular importance when Wnt signaling induces a polarized, asymmetric cell division.

\section{Materials and methods}

Genetics

The Hawaiian CB4856-derived lin-11(ne832) egg-laying deficient strain (WM59) was used as a starting strain. L4 animals mutagenized either with EMS or ENU were grown at $15^{\circ} \mathrm{C}$. $\mathrm{F} 2$ progeny were shifted to $25^{\circ} \mathrm{C}$ at the L4 stage for $36 \mathrm{~h}$, then down-shifted to $15^{\circ} \mathrm{C}$ for an additional $18 \mathrm{~h}$. A brief treatment with hypochlorite was then used to kill the adults and any hatched larvae, without dissolving away the adult cuticles. After a further $12-24 \mathrm{~h}$ of incubation at $15^{\circ} \mathrm{C}$, the carcasses were floated in a sucrose gradient to enrich for those that are less dense due to accumulated unhatched eggs within the body. Worm carcasses that float in the gradient were recovered and washed with M9. Candidate ts-mutant strains containing a few viable larvae (produced during the $18 \mathrm{~h}$ at $15^{\circ}$ ) and numerous inviable eggs produced at $25^{\circ} \mathrm{C}$ were selected under the dissecting microscope. Approximately one-fourth of the candidates proved to be temperature-sensitive embryonic-lethal mutants.

The strain WM75 contains the wrm-1(tm514) 423 base pair deletion allele provided by Dr. Shohei Mitani of the National Bioresource Project in Japan, and an integrated rescuing GFP-tagged wrm-1 transgene, neIs2, constructed by homologous recombination in yeast, as previously described (Rocheleau et al. 1997). C. elegans culture and genetics were as described previously (Brenner 1974).

\section{RNAi assays}

RNAi was performed as previously described (Bei et al. 2002). For ranrelated molecules, dsRNA of the entire coding region of ran-1/Ran, ran3/RCC1, and ran-5/RanBP3 were used. RNAi targeting, PAR-5/14-3-3, which is required for POP-1 nuclear export (Lo et al. 2004), failed to alter WRM-1::GFP localization.

\section{Live embryo imaging}

WRM-1::GFP embryos were mounted in M9 buffer on RITE-ON glass slides (Becton Dickinson), covered with a coverslip, and sealed with Vaseline. DIC and GFP images were captured at 1- to 2-min intervals using a Zeiss Axioplan2 Microscope and OPENLAB software.

\section{Acknowledgments}

We are grateful to members of our laboratory and Y. Yoneda for helpful discussion, and to H. Sawa for sharing unpublished data and materials. 
We thank C. elegans Genetic Center and National Bioresource Project in Japan for strains.

\section{References}

Bei, Y., Hogan, J., Berkowitz, L.A., Soto, M., Rocheleau, C.E., Pang, K.M., Collins, J., and Craig, C.C. 2002. SRC-1 and Wnt signaling act together to specify endoderm and to control cleavage orientation in early C. elegans embryo. Dev. Cell 3: 113-125.

Bowerman, B. and Shelton, C.A. 1999. Cell polarity in the early C. elegans embryo. Curr. Opin. Genet. Dev. 9: 390-395.

Brenner, S. 1974. The genetics of Caenorhabditis elegans. Genetics 77: 71-94.

Bullions, L.C. and Levine, A.J. 1998. The role of $\beta$-catenin in cell adhesion, signal transduction and cancer. Curr. Opin. Oncol. 10: 81-87.

Cadigan, K.M. and Nusse, R. 1997. Wnt signaling: A common theme in animal development. Genes \& Dev. 11: 3286-3305.

Cong, F. and Varmus, H. 2004. Nuclear-cytoplasmic shuttling of Axin regulates subcellular localization of b-catenin. Proc. Natl. Acad. Sci. 101: 2882-2887.

Fornerod, M., Ohno, M., Yoshida, M., and Mattaj, I.W. 1997. CRM1 is an export receptor for leucine-rich nuclear export signals. Cell 90: 10511060.

Fukuda, M., Asano, S., Nakamura, T., Adachi, M., Yoshida, M., Yanagida, M., and Nishida, E. 1997. CRM1 is responsible for intracellular transport mediated by the nuclear export signal. Nature 390: 308-311.

Goldstein, B. 1993. Establishment of gut fate in the E lineage of C. elegans: The roles of lineage-dependent mechanisms and cell interactions. Development 118: 1267-1277.

Gong, Y., Mo, V., and Fraser, S.E. 2004. Planar cell polarity signalling controls cell division orientation during zebrafish gastrulation. $\mathrm{Na}$ ture 430: 689-693.

Inoue, T., Oz, H.S., Wiland, D., Gharib, S., Deshpande, R., Hill, R.J., Katz, W.S., and Sternberg, P.W. 2004. Cell 118: 795-806.

Ishitani, T., Ninomiya-Tsuji, J., Nagai, S., Nishita, M., Meneghini, M., Barker, N., Waterman, M., Bowerman, B., Clevers, H., Shibuya, H., et al. 1999. The TAK1-NLK-MAPK-related pathway antagonizes signaling between b-catenin and transcription factor TCF. Nature 399: 798-802.

Kaletta, T., Schnabel, H., and Schnabel, R. 1997. Binary specification of the embryonic lineage in Caenorhabditis elegans. Nature 390: 294298.

Korswagen, H.C., Coudreuse, D.Y.M., Betist, M.C., Water, S., Zivkovic, D., and Clevers, H.C. 2002. The Axin-like protein PRY-1 is a negative regulator of a canonical Wnt pathway in C. elegans. Genes \& Dev. 16: 1291-1302.

Lin, R., Hill, R.J., and Priess, J. 1998. POP-1 and anterior-posterior fate decisions in C. elegans embryos. Cell 92: 229-239.

Lo, M.-C., Gay, F., Odom, R., Shi, Y., and Lin, R. 2004. Phosphorylation by the $\beta$-catenin/MAPK complex promotes 14-3-3-mediated nuclear export of TCF/POP-1 in signal-responsive cells in C. elegans. Cell 117: 95-106.

Lu, W., Yamamoto, V., Ortega, B., and Baltimore, D. 2004. Mammalian Ryk is a Wnt coreceptor required for stimulation of neurite outgrowth. Cell 119: 97-108.

Lyuksyutova, A.I., Lu, C-C., Milanesio, N., King, L.A., Guo, N., Wang, Y., Nathans, J., Tessier-Lavigne, M., and Zou, Y. 2003. Anteriorposterior guidance of commissural axons by Wnt-frizzled signaling. Science 302: 1984-1988.

Maduro, M.F., Lin, R., and Rothman, J.H. 2002. Dynamics of developmental switch: Recursive intracellular and intranuclear redistribution of Caenorhabditis elegans POP-1 parallels Wnt-inhibited transcriptional repression. Dev. Biol. 248: 128-142.

Meneghini, M.D., Ishitani, T., Carter, J.C., Hisamoto N., NinomiyaTsuji, J., Thorpe, C.J., Hamill, D.R., Matsumoto, K., and Bowerman, B. 1999. MAP kinase and Wnt pathways converge to downregulate an HMG-domain repressor in Caenorhabditis elegans. Nature 399: 793797.

Nelson, J.W. and Nusse, R. 2004. Convergence of Wnt, $\beta$-catenin, and cadherin pathways. Science 303: 1483-1487.

Polakis, P. 2000. Wnt signaling and cancer. Genes \& Dev. 14: 1837-1851. Rocheleau, C.E., Downs, W.D., Lin, R., Wittmann, C., Bei, Y., Cha, Y.H., Ali, M., Priess J.R., and Mello, C.C. 1997. Wnt signaling and an APC- related gene specify endoderm in early C. elegans embryos. Cell 90: 707-716.

Rocheleau, C.E., Yasuda, J., Shin, T.H., Lin, R., Sawa, H., Okano, H., Priess J.R., Davis, R.J., and Mello, C.C. 1999. WRM-1 activates the LIT-1 protein kinase to transduce anterior/posterior polarity signals in C. elegans. Cell 97: 717-726.

Rosin-Arbesfeld, R., Cliffe, A., Brabletz, T., and Bienz, M. 2003. Nuclear export of the APC tumour suppressor controls b-catenin function in transcription $E M B O$ T. 22: 1101-1113.

Schlesinger, A., Shelton, C.A., Maloof, J.N., Meneghini, M., and Bowerman, B. 1999. Wnt pathway components orient a mitotic spindle in the early Caenorhabditis elegans embryo without requiring gene transcription in the responding cell. Genes \& Dev. 13: 2028-2038.

Shin, T.H., Yasuda, J., Rocheleau, C.E., Lin, R., Soto, M., Bei, Y., Davis, R.J., and Mello, C.C. 1999. MOM-4, a MAP kinase kinase kinaserelated protein, activates WRM-1/LIT-1 kinase to transduce anterior/ posterior polarity signals in C. elegans. Mol. Cell 4: 275-280.

Takeshita H. and Sawa H. 2005. Asymmetric cortical and nuclear localizations of WRM-1/ $\beta$-catenin during asymmetric cell division in $C$. elegans. Genes \& Dev. (this issue).

Thorpe, C.J., Schlesinger, A., Carter, J.C., and Bowerman, B. 1997. Wnt signaling polarizes an early $C$. elegans blastomere to distinguish endoderm from mesoderm. Cell 90: 695-705.

Tolwinski, N.S. and Wieschaus, E. 2001. Armadillo nuclear import is regulated by cytoplasmic anchor Axin and nuclear anchor dTCF/Pan. Development 128: 2107-2117.

E95.

Townsley, F.M., Cliffe, A., and Bienz, M. 2004. Pygopus and Legless target Armadillo/ $\beta$-catenin to the nucleus to enable its transcriptional co-activator function. Nat. Cell Biol. 6: 626-633.

Willert, K. and Nusse, R. 1998. $\beta$-Catenin: A key mediator of Wnt signaling. Curr. Opin. Genet. Dev. 8: 95-102.

Yoshikawa, S., McKinnon, R.D., Kokel, M., and Thomas, J.B. 2003. Wntmediated axon guidance via the Drosophila Derailed receptor. Nature 422: $583-588$.

Zou, Y. 2004. Wnt signaling in axon guidance. Trends Neurosci. 27: 528 532. 


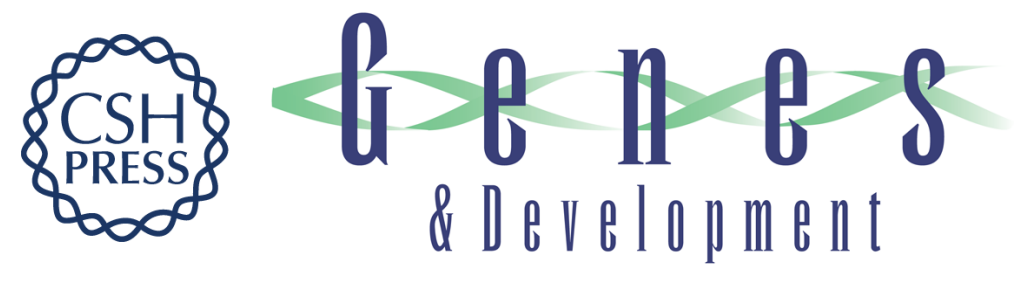

\section{Wnt signaling drives WRM-1/ $\beta$-catenin asymmetries in early $C$. elegans embryos}

Kuniaki Nakamura, Soyoung Kim, Takao Ishidate, et al.

Genes Dev. 2005, 19:

Access the most recent version at doi:10.1101/gad.1323705

\section{Supplemental http://genesdev.cshlp.org/content/suppl/2005/12/16/19.15.1749.DC1 Material}

References This article cites 34 articles, 11 of which can be accessed free at: http://genesdev.cshlp.org/content/19/15/1749.full.html\#ref-list-1

\section{License}

Email Alerting

Receive free email alerts when new articles cite this article - sign up in the box at the top Service 